\title{
La influencia de los roles de género en el consumo de alcohol: estudio cualitativo en adolescentes y jóvenes en Asturias
} The influence of gender roles in alcohol consumption: a
qualitative study of adolescents and young adults in Asturias

\author{
María Aránzazu Fernández Rodríguez*, Sandra Dema Moreno**, Yolanda Fontanil Gómez***. \\ * Doctoranda del Programa "Género y Diversidad" de la Universidad de Oviedo y Directora del Área de Prevención de la \\ Fundación C.E.S.P.A.-Proyecto Hombre. ** Profesora Titular de Sociología de la Universidad de Oviedo. *** Profesora Titular \\ de Psicología de la Universidad de Oviedo.
}

\section{Resumen}

A pesar del desarrollo de políticas de prevención dirigidas a abordar el consumo de alcohol en adolescentes y jóvenes, durante la última década se ha producido un aumento considerable de quienes realizan un consumo abusivo de esta sustancia en España. Las encuestas oficiales sobre este fenómeno muestran un acercamiento entre varones y mujeres en las prevalencias de consumo y en los consumos de riesgo, e incluso una mayor incidencia de estas conductas en el caso de las mujeres al final de la etapa adolescente.

El objetivo de este artículo es conocer la influencia que ejercen los roles de género en estas pautas de consumo en adolescentes y jóvenes. Para ello, se han realizado grupos focales que han permitido identificar las similitudes y diferencias que se producen tanto en las prácticas de consumo y abuso del alcohol que desarrollan estos colectivos, como en el significado social que unos y otras atribuyen a las citadas prácticas.

Los resultados obtenidos muestran que las variables género y edad actúan de forma combinada en el aprendizaje del consumo de bebidas alcohólicas, así como en las motivaciones y expectativas que los y las adolescentes y jóvenes tienen sobre dichas prácticas. Además, en este estudio se identifican tres etapas diferenciadas, en la primera de ellas los roles de género se encuentran claramente definidos, en la segunda se observa cierta transgresión de los mismos fundamentalmente por parte de las mujeres y en la última se advierte una vuelta a los tradicionales roles de género.

Palabras clave: Género; Alcohol; Adolescencia; Juventud; Investigación cualitativa.

\begin{abstract}
Despite the implementation of prevention policies aimed at addressing alcohol consumption among both adolescents and young adults, there has been a considerable increase in those who abuse alcohol in Spain over the last decade. Official surveys on this phenomenon show that both the prevalence and risky consumption of men and women are reaching similar levels, with even higher figures for these behaviours in the case of girls at the end of adolescence.

The aim of this article is to understand the influence of gender roles in the consumption of alcohol among adolescents and young adults. To this end, focus groups have been employed to identify similarities and differences both in drinking patterns and alcohol abuse among young males and females, as well as in the social meaning that both groups attribute to these practices.

The results obtained show that the variables gender and age act in a combined way on the learning of alcohol consumption, as well as on the motivations and expectations that adolescents and young adults have regarding these practices. In addition, in this study three differentiated stages are identified: in the first, gender roles are clearly defined; in the second, there is a certain transgression of these roles mainly by young women, and in the third, there is a return to traditional gender roles.

Keywords: Gender; Alcohol; Adolescence; Young adult; Qualitative research.
\end{abstract}




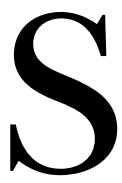
egún el Informe Mundial de situación sobre Alcohol y Salud 2014, elaborado por la Organización Mundial de la Salud, el uso nocivo ${ }^{1}$ de alcohol causa 3,3 millones de muertes al año, lo que representa el $5,9 \%$ de las defunciones del año 2012. En este Informe también se señalan diferencias significativas en relación con el sexo, el 7,6\% de las muertes de varones fueron atribuidas al alcohol, mientras que en el caso de las mujeres este porcentaje baja al 4\% (Organización Mundial de la Salud, 2014). Una parte considerable de las defunciones corresponde a personas jóvenes, concretamente el $25 \%$ de las muertes entre 20 a 39 años fueron atribuidas al consumo de esta sustancia. Asimismo, este tipo de consumo abusivo incrementa el riesgo de padecer determinadas enfermedades físicas (trastornos cardiovasculares, cirrosis, etc.) y mentales (ansiedad, depresión, etc.), además de aumentar la probabilidad de sufrir algún tipo de accidente o de involucrarse en actos violentos. No en vano, el uso abusivo de bebidas alcohólicas ocupa el tercer lugar entre los principales riesgos para la salud en el mundo, constituyendo un problema importante de salud pública, con sustanciales costes sociosanitarios para la propia persona que lo realiza, para quienes la rodean y para la sociedad en general.

En nuestro país, la última Encuesta sobre Uso de Drogas en Enseñanzas Secundarias (ESTUDES), realizada por el Observatorio Español de la Droga y la Toxicomanía (OEDT), en 2014, señala que el alcohol es, con diferencia, la sustancia más consumida entre la población escolar de 14 a 18 años. Así, el 78,9\% de la población adolescente reconoce haber consumido alcohol alguna vez en su vida, siendo dicho consumo ligeramente superior entre las mujeres, con independencia del tramo temporal que se considere.

La citada Encuesta sitúa la edad de inicio en el consumo de alcohol en la temprana edad de 13,8 años, con datos muy similares para los y las adolescentes, 13,8 y 13,9 años respectivamente.

En cuanto al uso abusivo de esta sustancia, una de cada cuatro personas de 14 años relata haber sufrido una intoxicación etílica alguna vez en su vida, a los 16 años el porcentaje asciende al $56,5 \%$ y a los 18 años al $74,1 \%$. Desde hace al menos una década, las adolescentes admiten haberse emborrachado alguna vez en la vida en mayor proporción que los varones $(51,4 \%$ de las chicas frente al $48,7 \%$ de los chicos).

Una vez superada la mayoría de edad, aparecen cambios en las prevalencias y los patrones de consumo de alcohol condicionados por la edad y el sexo. Por un lado, deja de concentrarse la citada práctica en el fin de semana, y es que a medida que aumenta la edad se extiende su uso a los

1 Término introducido por la Estrategia Mundial para Reducir el Uso Nocivo de Alcohol, referido sólo a los efectos del consumo de alcohol en la salud pública, sin perjuicio alguno de las creencias religiosas y las normas culturales (OMS, 2010). días de diario, siendo esta cuestión especialmente significativa en el caso de los varones. La última Encuesta sobre Alcohol y Drogas en España (EDADES 2015-2016) señala que el consumo diario de alcohol es 3,5 veces superior en los hombres en relación con las mujeres. Por otro lado, comienza a apreciarse en los varones mayores prevalencias relacionadas con el abuso de dicha sustancia (intoxicaciones etílicas y binge drinking) en relación con las mujeres, a pesar de registrarse un descenso en las prevalencias de este tipo de prácticas a medida que aumenta la edad. Por ejemplo, en el caso del binge drinking, hay una diferencia de aproximadamente 5 puntos porcentuales en el segmento de edad de 15 a 19 años, mientras que en el de 20 a 24 años esa diferencia aumenta hasta los 10 puntos aproximadamente, y a partir de esa edad, el porcentaje de varones que realiza binge drinking duplica al de mujeres.

La incorporación de la perspectiva de género en el estudio del consumo de alcohol en adolescentes y jóvenes es relativamente reciente, particularmente en nuestro país. Las investigaciones realizadas desde un enfoque cuantitativo se han centrado en identificar las diferencias de género en el consumo de alcohol en estos grupos de edad (Galán, González y Valencia-Martín, 2014; Colell, Sánchez-Niubò y Domingo-Salvany, 2013; Roberts, 2012; Emslie, Lewars, Batty y Hunt, 2009; Delgado, Bautista, Inglés, Espada y Torregrosa, 2005), destacando entre ellas las que abordan las motivaciones y las consecuencias sobre la salud asociadas a un consumo abusivo (Bousoño Serrano et al., 2017; Díaz-Mesa et al., 2016; Secades Villa, López Núñez, Fernández Artamendi, Weidberg y Fernández Hermida, 2013; Measham y Østergaard, 2009; De la Villa Moral Jiménez, Rodríguez Díaz y Sirvent Ruiz, 2005).

Las investigaciones de carácter cualitativo, por su parte, han tratado de comprender el significado del consumo abusivo de alcohol en adolescentes y jóvenes, apuntando que el inicio de dicha conducta está ligado a la consideración de la misma como algo característico y normativo de esta etapa (Davies, Martin y Foxcroft, 2013), unido al proceso de adquisición de madurez (Romo Avilés, Marcos Marcos, Gil García, Marquina Márquez y Tarragona Camacho, 2015; Ortiz García y Clavero Díaz, 2014). Por lo tanto, no es de extrañar la incorporación de las mujeres a esta práctica durante la última década, ya que cada vez en mayor medida, identifican el alcohol como una parte constitutiva de su vida social, que posibilita la incorporación a espacios de ocio y prácticas tradicionalmente masculinas (Gómez Moya, Arnal Gómez, Martínez Vilanova y Muñoz Rodríguez, 2010).

Esta convergencia entre chicos y chicas respecto al consumo de alcohol ha sido considerada como una forma de romper los códigos tradicionales de género por parte de las mujeres (Romo Avilés et al., 2015). Desde algunas posiciones feministas, el consumo público de alcohol ha sido interpretado como un reflejo de la dominación masculina en los espacios públicos y de ocio, por lo que la mayor pre- 
sencia femenina en este tipo de espacios y la adopción por parte de las mujeres de dichas conductas asociadas tradicionalmente a los varones, supondría el fin de tal dominio. Sin embargo, en esta transgresión de los roles de género no se debe obviar el potencial peligro al que se exponen las mujeres al llevar a cabo estas conductas de riesgo (Rolfe, Orford y Dalton, 2009).

La bibliografía especializada nos muestra que el consumo de alcohol es una actividad influenciada por el género, pero los roles de género no son estáticos. La redefinición de los mismos y la influencia del consumo de alcohol ha sido analizada por algunas investigaciones que han llegado a la conclusión de que los cambios en los patrones normativos de consumo de esta sustancia revelan importantes rupturas de género en la adolescencia (Romo Avilés et al., 2015; Romo Avilés, Meneses Falcón y Gil García, 2014; Gómez Moya et al., 2010). Sin embargo, tales investigaciones no suelen diferenciar cómo se producen dichas rupturas de género en las distintas etapas de la adolescencia y primera juventud, momentos en los que la información proporcionada por las principales encuestas indica que se inicia el consumo de alcohol y se llevan a cabo las conductas de mayor riesgo (intoxicaciones etílicas y/o binge drinking).

En este artículo, basado en una metodología de carácter cualitativo, se ha querido profundizar en esta cuestión, analizando las experiencias, percepciones y significados que las personas adolescentes y jóvenes atribuyen al consumo y abuso del alcohol en tres cohortes de edad diferentes: 13-15 años, 16-18 años y 19-24 años. El objetivo consiste en conocer cómo influyen los roles de género en el consumo de alcohol en estos grupos de población, identificando las similitudes y diferencias de género en las prácticas que desarrollan y en las razones que esgrimen a la hora de sustentar las citadas prácticas. Todo ello con vistas a incrementar el conocimiento actual sobre cómo se da el proceso de inicio en el consumo de alcohol por parte de varones y mujeres y cómo se perfilan las pautas de consumo durante la juventud y su conexión con la vida adulta.

\section{Método}

Para la realización de la investigación empleada en la elaboración de este artículo se utilizó una metodología de carácter cualitativo consistente en la realización y análisis de seis grupos focales a personas adolescentes y jóvenes. Esta técnica de recogida de información supuso un acierto, dado que favorece las interacciones entre las personas que componen el propio grupo lo que, por un lado, permite profundizar en el conocimiento de las experiencias vitales de adolescentes y jóvenes en relación al consumo de alcohol y, por otro, analizar la influencia del contexto social y cultural en el significado de sus conductas.

La selección de las personas participantes en los grupos focales se realizó en función de las siguientes variables: sexo, edad, diversidad socioeconómica y nivel educativo, siguiendo los criterios expuestos por Sánchez Gómez (2004): I) muestreo intencional y razonado, II) selección de informantes que puedan explicar mejor el fenómeno estudiado, III) muestreo acumulativo y secuencial hasta conseguir la saturación del discurso, IV) información exhaustiva, rica en matices, profunda y completa. En dicha selección se ha intentado garantizar a la vez la necesaria homogeneidad para que en los citados grupos se pudiera producir un discurso colectivo más allá del individual, así como la heterogeneidad que permitiera contar con los diversos discursos sociales existentes.

El trabajo de campo se realizó entre los meses de marzo a mayo de 2013 en el Concejo de Oviedo, capital del Principado de Asturias, comunidad autónoma del norte de España. Siguiendo los criterios de selección mencionados anteriormente, se llevaron a cabo seis grupos focales, compuestos por adolescentes y jóvenes, la mitad de ellos del sexo femenino, y la otra mitad del sexo masculino. Se consideró pertinente esta distribución para facilitar que el discurso fluyera de manera libre y no se viera condicionado por la presencia de personas del otro sexo en el grupo. Especialmente, debido a que pensamos, como así sucedió, que en los grupos podrían surgir cuestiones relacionadas con sexualidad, violencia, estereotipos y roles de género $\mathrm{y} / \mathrm{u}$ otros temas acerca de los cuales pueden existir no solo vivencias diferentes por parte de varones y mujeres, sino incluso discursos enfrentados que habrían dificultado la propia dinámica grupal. Además, y atendiendo a la edad, se establecieron tres cohortes diferenciadas por criterios evolutivos en torno a la madurez cognitiva y emocional presente en cada una de ellas: 13-15 años, 16-18 años y 1924 años.

En lo que se refiere a la diversidad socioeconómica se valoró la utilización de criterios habituales tales como el nivel de estudios, renta y profesión de sus progenitores, pero dado que dicha información resultaba difícil de recabar en la fase de selección de las potenciales personas integrantes de los grupos optamos como alternativa por la combinación del lugar de residencia y la titularidad pública, privada o concertada del centro educativo de quienes se encontraban cursando Enseñanza Secundaria Obligatoria o Bachillerato. Así, se seleccionaron personas cuya residencia estuviera fijada en distintas zonas del Concejo de Oviedo: centro, periferia, rural, y URBAN². Oviedo se caracteriza por ser una ciudad que se ha desarrollado territorialmente de forma concéntrica. En el centro de la ciudad se localiza mayoritariamente la población con un nivel socioeconómico alto, procedente en buena medida de la

2 Esta zona identifica a barrios o espacios en los que en el momento de la investigación se estaban llevando a cabo actuaciones de regeneración económica, medioambiental y social a través de un proyecto URBAN cofinanciado por el Fondo Europeo de Desarrollo Regional (FEDER). 
burguesía regional, cuyo poder adquisitivo se refleja especialmente en aquellas familias cuyos descendientes acuden a centros privados y en menor medida a los concertados. En los barrios de la periferia encontramos fundamentalmente familias de origen obrero y extracto social medio y medio bajo, mientras que la zona URBAN habita la población desfavorecida, con un fuerte índice de desempleo, menor nivel educativo, débil tasa de actividad económica y alto nivel de pobreza y exclusión en comparación con el resto de la ciudad (Ayuntamiento de Oviedo, 2008). En los grupos focales incorporamos también personas que viven en la zona rural del concejo, por las características particulares de este entorno geográfico, sin embargo, no se ha encontrado un discurso diferenciado en función de la procedencia urbana o rural de adolescentes y jóvenes. Para garantizar la diversidad en cuanto a nivel educativo y/o cultural, en los grupos de edad en los que existían varias opciones, se seleccionaron personas de todos los itinerarios formativos posibles: Bachillerato, Formación Profesional, ciclos formativos de grado medio o superior y estudios universitarios. Asimismo, en los grupos de discusión no solo se incorporaron personas que se encontraban desarrollando sus estudios, sino también en lo posible trabajadoras y en situación de desempleo.

Señalar, finalmente, que el criterio principal de inclusión para la selección de la muestra fue que las personas seleccionadas realizasen un consumo de alcohol en su tiempo de ocio, o perteneciesen a un grupo de iguales en el que existiese consumo de alcohol asociado al ocio.
Tal como se recoge en la Tabla 1, la muestra está formada por 44 adolescentes y jóvenes, 23 mujeres y 21 hombres, con edades comprendidas entre los 13 y los 24 años. Para la captación de estas personas se contó con la colaboración de profesionales que de manera habitual desarrollan su trabajo con esta población: equipos directivos y profesorado de centros educativos y escuelas taller, entidades del ámbito de la intervención social, y diferentes servicios y programas municipales. Además, y para conseguir la participación de personas con determinados perfiles, se utilizó la técnica de bola de nieve, si bien al tratarse de una muestra de carácter cualitativo, en modo alguno se pretendía que fuera representativa de la población.

Los grupos focales fueron moderados por una socióloga y dos psicólogas debidamente entrenadas. La secuencia general del desarrollo de los grupos fue: presentación de la investigación, estímulo inicial dirigido a generar la discusión, estímulos secundarios dirigidos a orientarla cuando esta no transcurría de forma natural y/o precisaba ser reconducida, y estímulo final. La duración aproximada de cada grupo focal osciló entre 60 y 90 minutos.

Los grupos focales fueron grabados y transcritos, para posteriormente ser codificados y analizados con el programa informático ATLAS. Ti versión 7. Para obtener una mayor fiabilidad en este proceso cada una de las investigadoras realizó una codificación por separado de los grupos focales, que se puso en común en sucesivas reuniones con el resto del equipo, dando lugar a un listado común de códigos (Tabla 2). A partir de los citados códigos se procedió

Tabla 1. Composición de los grupos focales y variables sociodemográficas de las personas seleccionadas

\begin{tabular}{|c|c|c|c|c|c|c|c|}
\hline Código grupo & № Participantes & Sexo & Edad & Zona resic & cia & $\begin{array}{c}\text { Estudios y tipo } \\
\text { de centro educativo }\end{array}$ & \\
\hline GF01 & 8 & Mujeres & 13-15 años & $\begin{array}{l}\text { Centro } \\
\text { Periferia } \\
\text { URBAN } \\
\text { Rural }\end{array}$ & $\begin{array}{l}2 \\
3 \\
1 \\
2\end{array}$ & $\begin{array}{l}\text { ESO Público } \\
\text { ESO Concertado }\end{array}$ & $\begin{array}{l}4 \\
4\end{array}$ \\
\hline GF02 & 8 & Hombres & 13-15 años & $\begin{array}{l}\text { Centro } \\
\text { Periferia } \\
\text { URBAN } \\
\text { Rural }\end{array}$ & $\begin{array}{l}2 \\
5 \\
1 \\
-\end{array}$ & $\begin{array}{l}\text { ESO Público } \\
\text { ESO Concertado }\end{array}$ & $\begin{array}{l}6 \\
2\end{array}$ \\
\hline GF03 & 10 & Mujeres & 16-18 años & $\begin{array}{l}\text { Centro } \\
\text { Periferia } \\
\text { URBAN } \\
\text { Rural }\end{array}$ & $\begin{array}{l}2 \\
4 \\
3 \\
1\end{array}$ & $\begin{array}{l}\text { Bachillerato Público } \\
\text { Bachillerato Concertado }\end{array}$ & $\begin{array}{l}7 \\
3\end{array}$ \\
\hline GF04 & 5 & Hombres & 16-18 años & $\begin{array}{l}\text { Centro } \\
\text { Periferia } \\
\text { URBAN } \\
\text { Rural }\end{array}$ & $\begin{array}{l}1 \\
2 \\
1 \\
1\end{array}$ & $\begin{array}{l}\text { Bachillerato Público } \\
\text { Bachillerato Concertado }\end{array}$ & $\begin{array}{l}3 \\
3\end{array}$ \\
\hline GF05 & 5 & Mujeres & 19-24 años & $\begin{array}{l}\text { Centro } \\
\text { Periferia } \\
\text { URBAN } \\
\text { Rural }\end{array}$ & $\begin{array}{l}2 \\
1 \\
1 \\
1\end{array}$ & $\begin{array}{l}\text { Escuela Taller } \\
\text { Universitario/a } \\
\text { Ldo./a Dpdo./a }\end{array}$ & $\begin{array}{l}1 \\
3 \\
1\end{array}$ \\
\hline GF06 & 8 & Hombres & 19-24 años & $\begin{array}{l}\text { Centro } \\
\text { Periferia } \\
\text { URBAN } \\
\text { Rural }\end{array}$ & $\begin{array}{l}1 \\
3 \\
4 \\
-\end{array}$ & $\begin{array}{l}\text { Bachillerato } \\
\text { Escuela Taller } \\
\text { FP } \\
\text { Universitario/a } \\
\text { Ldo./a Dpdo./a }\end{array}$ & $\begin{array}{l}1 \\
3 \\
1 \\
2 \\
1\end{array}$ \\
\hline
\end{tabular}


Tabla 2. Listado de códigos

\begin{tabular}{|c|c|}
\hline Códigos & Subcódigos \\
\hline Rutina de fin de semana asociada al consumo de alcohol & $\begin{array}{l}\text { Día/s de salir } \\
\text { Frecuencia } \\
\text { Zona } \\
\text { Hora } \\
\text { Cómo llegar a la zona de salir y cómo volver a casa }\end{array}$ \\
\hline \multicolumn{2}{|l|}{ Estrategias para convencer a padres y madres a la hora de salir } \\
\hline Tipo de consumo de alcohol & $\begin{array}{l}\text { Dónde se consume } \\
\text { Estrategias para consumir } \\
\text { Elección de bar } \\
\text { Botellón } \\
\text { Motivos para el consumo de alcohol } \\
\text { Imagen de uno mismo, reputación atribuida }\end{array}$ \\
\hline \multicolumn{2}{|l|}{ Estrategias de reducción de riesgos } \\
\hline Percepción seguridad ciudadana & $\begin{array}{l}\text { Intervención policial } \\
\text { Peleas }\end{array}$ \\
\hline \multicolumn{2}{|c|}{ Utilización de redes sociales durante el consumo de alcohol y/o después del mismo } \\
\hline \multicolumn{2}{|l|}{ Comunicación intrafamiliar sobre el consumo de alcohol } \\
\hline Programas de formación/información & $\begin{array}{l}\text { Eficacia } \\
\text { Propuestas }\end{array}$ \\
\hline Género & $\begin{array}{l}\text { Identidad de género } \\
\text { Roles de género } \\
\text { Estereotipos de género } \\
\text { Diferencias/similitudes de género }\end{array}$ \\
\hline
\end{tabular}

al análisis de cada uno de los fragmentos codificados, que nuevamente se desarrolló de la misma forma, en un primer momento, por cada una de las investigadoras individualmente y posteriormente puesto en común en el equipo, hasta lograr una interpretación consensuada, realizando de esta manera una triangulación de los resultados obtenidos mediante el análisis independiente y el contrastado por las tres autoras de este artículo.

Esta investigación se ha llevado a cabo según los principios de deontología profesional exigibles en el trato con personas, tales como la garantía de confidencialidad y anonimato. Además, se han seguido los preceptos éticos que regulan el trabajo con adolescentes, por lo que se informó a los padres y madres de los y las adolescentes participantes sobre las características de la investigación, los objetivos abordados, el método utilizado y se requirió por escrito su consiguiente consentimiento.

\section{Resultados}

\section{Etapa 1: La influencia de las relaciones de género en el inicio del consumo de alcohol}

En los grupos focales de las personas entre 13 y 15 años de edad aparece narrado con detalle el proceso de acercamiento al consumo de alcohol, coincidiendo los discursos de ambos sexos en señalar a varones de mayor edad como la figura de referencia en este rito de inicio, y en ocasiones, quienes les facilitan el acceso a las bebidas alcohólicas, sobre todo en el caso de las chicas.

En el siguiente fragmento, los adolescentes de 13 a 15 años relatan cómo otros varones, ya sean amigos o familiares, les introducen en el consumo de alcohol. Y como indican algunos de ellos, sus primeros consumos de alcohol los realizan incluso en compañía de sus progenitores varones:

Preceptora: ¿En el momento en que probasteis el alcohol, con quién estabais?

Chico 1: Con amigos.

Chico 2: Con mi hermano.

Chico 1: Con amigos, qué ejemplo ¿eh?

Chico 3: Sí, con los amigos, siempre.

Chico 4: Con los amigos y hay veces que hasta mis padres o familiares te dan a probar.

Chico 5: Yo siempre bebo con los amigos, nunca bebo solo. Con los amigos los sábados y eso.

Chico 6: Yo la primera vez con mi padre, la segunda con mi padre, la tercera aún no. (GF02. Chicos 13-15 años)

Salvando el comentario irónico del participante 1, ese rol de iniciadores está totalmente asumido por el grupo, como corresponde a una conducta socialmente aceptada. 
La función que cumplen los citados varones, además de acompañar el inicio en el consumo de alcohol, consiste en facilitar el aprendizaje de toda una serie de estrategias dirigidas a gestionar dicho consumo, tanto para evitar situaciones relacionadas con una ingesta abusiva, como para afrontarlas una vez que ocurren:

Chico 3: Pues lo que te dicen tus amigos.

Chico 1: Eso, lo que te dicen ellos. Yo aprendo de ellos estos trucos, yo no tenía ni idea.

Chico 4: Yo hay veces que pregunto a mis padres, sobre todo a mi padre, me aconsejan qué puedo hacer y demás. (GF02. Chicos 13-15 años)

Las adolescentes de 13 a 15 años, por su parte, relatan igualmente que dicho proceso lo realizan de la mano de chicos algo mayores que ellas, que ya tienen un consumo ocasional de alcohol y, por tanto, más experiencia:

Chica 1: Nosotras, hay gente que puede ser más mayor que tú y que ya haya salido más veces y que te enseña los trucos que hay para no emborracharte tanto: no mezclar, no... Siempre hay pequeños trucos que te van enseñando... y qué bares son más adecuados que otros que no son tan buenos. Pues que a lo mejor te dicen que no vayas por esta zona porque se venden cosas ilegales o... estar en zonas iluminadas, y... o sea, nunca os emborrachéis mucho porque ya se sabe lo que pasa... no dejar la copa por ahí. Tapar siempre el vaso... (GF01. Chicas 13-15 años)

Esta enseñanza, como se puede observar, está impregnada de los roles de género asignados socialmente. Se advierte a las adolescentes sobre qué tipo de ambientes son los más adecuados para ellas, así como sobre las posibles agresiones que pueden sufrir en estos contextos, junto con la recomendación de que no beban demasiado y estén alertas en todo momento ante lo que les pudiera suceder.

Estos varones, que suelen ser novios de alguna de las chicas del grupo o hermanos mayores, no solo enseñan cómo consumir y gestionar los riesgos asociados a este consumo, sino que acompañan y protegen a las adolescentes durante dicho aprendizaje:

Chica 2: ... el novio de una amiga nuestra es dos años mayor que nosotras entonces ya salió más y cuando salimos pues viene él con nosotras y sí nos lo dice que tengamos cuidado. (GF01. Chicas 13-15 años)

(...)

Chica 3: Ah, bueno, sí que hay gente que utiliza a los mayores, o sea mayores de edad para eso, para que te compre no sé qué o para que me defiendas que quiero ir a no sé dónde o que finjas que eres mi hermano, cosas así. O sea, que hay una amiga que tiene un amigo de su novio, o algo así, que es mayor de edad $y$, entonces, claro, gracias a él ella consigue, o sea, el novio puede ir a bares que no son para menores... O sea, como que están compinchados con otra gente y consiguen más de lo que podrían conseguir. (GF01. Chicas 13-15 años)

Tal y como podemos observar en estos dos relatos, para las adolescentes los chicos cumplen una clara función en el inicio del consumo de alcohol. Son quienes facilitan el acercamiento de las chicas a los contextos de consumo y el acceso a la sustancia. Pero también juegan un importante papel en lo relativo al aprendizaje del ritual de consumo y a la protección de las jóvenes ante los riesgos y peligros que pueden sufrir en los bares y otros espacios de ocio semejantes.

En contraposición a esta figura, las personas de edades más jóvenes hacen referencia en sus discursos a otra que se encarga de velar por quienes se encuentran bajo los efectos del consumo abusivo de alcohol. Este rol puede ser ejercido tanto por un chico como por una chica del grupo, pero establecen importantes diferencias de género respecto al valor y significado que se da a esa figura.

En los grupos de las adolescentes, cuando ese rol protector es ejercido por un varón se le atribuye una función de control, generalmente de un chico hacia su novia, como se puede apreciar en el siguiente relato:

Chica 2: Sí, nosotras y el novio de nuestra amiga somos los que controlamos al resto del grupo. Él porque quiere controlar a su novia y nos ayuda a controlar. (GF01. Chicas 13-15 años)

Por el contrario, cuando el rol es asumido por una mujer lo perciben vinculado a la labor de cuidado e inmediatamente establecen una asociación entre cuidado y maternidad, que además es devaluada por el grupo, tachando a las adolescentes que ejercen este cuidado de pesadas:

Chica 2: Sí, las que normalmente no bebemos casi nada nos llaman pesadas porque estamos: "Para de beber".

Chica 3: "Para de beber", porque luego te estás cayendo, luego no te acuerdas, luego la resaca y... no nos hacen caso. Luego te dicen lo típico de: "Sí, mami". (GF01. Chicas 13-15 años)

Curiosamente, cuando esta misma conducta es desarrollada por un varón en el grupo de chicos más jóvenes, a diferencia de lo que ocurre en el grupo de las chicas, este rol de cuidado no aparece devaluado por el resto de integrantes, sino que es demandado por ellos y además valorado tanto por el grupo como por quien lo ejerce de manera positiva: 
Chico 4: Era yo y sigo siendo yo. Es que hasta te lo ponen ellos: "tú, que no bebes mucho, por favor, ayúdame por si me paso". O si no, yo lo hago tranquilamente y me siento bien en hacerlo. Estás cuidando entre comillas y ayudándoles a que no cometan errores. (GF02. Chicos 13-15 años)

\section{Etapa 2: La generalización de los consumos de riesgo de alcohol en la adolescencia y la adscripción/ruptura con los roles de género asignados}

Los relatos de los y las adolescentes de entre 16 y 18 años describen que el consumo abusivo de alcohol es una práctica habitual y normalizada, vinculada al ocio de fin de semana. En sus discursos, no aparecen diferencias de género relativas a prevalencias y pautas de consumo, pero sí en cuanto a la forma de actuar de unos y otras tras una ingesta abusiva de alcohol y en el significado que unas y otros atribuyen a sus acciones, observándose en esta etapa una cierta trasgresión de los roles de género, sobre todo en el caso de las adolescentes.

Varones y mujeres identifican la expresión exagerada de las emociones como conducta típica femenina tras la ingesta abusiva de alcohol. Pero mientras que los discursos de las adolescentes inciden en la exaltación del afecto, los de los ellos, por el contrario, hacen referencia al llanto femenino, como se puede observar en los siguientes dos fragmentos:

Chica 2: Sí, pero eso, los chicos, ¿eh? Hay chicos que cuando beben, en general, pues algunos que se mantienen que parece que no han bebido y otros que sí. Pero, sin embargo, las chicas siempre que beben, lo manifiestan y se nota mucho más.

Chica 3: Estamos más cariñosas las chicas. Yo, por lo menos soy más... un abrazo.

Chica 2: Pueden estar muy borrachos y tal y no lo notas. En algunas personas. En otras sí, por supuesto. Pero las chicas, yo creo que siempre se les nota.

Chica 3: Sí.

Chica 2: Porque como que son mucho más expresivas. Chica 3: Muchísimo más. (GF03. Chicas 16-18 años)

Chico 3: Las chicas, la mitad de ellas acaban llorando. Por pijadas.

Chico 1: Veo una fila de chicas llorando, una empieza a llorar y la otra llora porque llora ella.

Chico 2: Sí.

Chico 3: Todas llorando.

Chico 1: Sí. (GF04. Chicos 16-18 años)

Llama la atención lo estereotipado de ambos discursos, que vinculan con claridad la exteriorización de las emociones con las mujeres, frente a los varones, que o bien resisten la ingesta abusiva de bebidas alcohólicas sin resultar afectado su comportamiento o, como se muestra a continuación, se expresan de forma agresiva. Pero como se decía anteriormente, mientras que las adolescentes ponen el acento en que ellas expresan emociones positivas, como el afecto, los varones por el contrario perciben que las mujeres cuando beben en exceso lloran, ridiculizando esta conducta que entienden como grupal e injustificada. Por un lado, destaca la vertiente irracional e infundada de la conducta femenina, lo hacen como dice el participante 3 "por pijadas". Y, por otro, la interpretan como si fuera una actuación casi contagiosa, es decir, inevitable, que sucede por la presión del grupo y que anula la capacidad individual de cada una de las adolescentes, que se dejarían llevar por la vertiente emocional.

Respecto a la conducta de los adolescentes se percibe más variedad. Ellas señalan que cuando los chicos beben más de la cuenta o bien no se les nota, como afirma la participante 2, o bien desarrollan conductas agresivas.

Por su parte, la visión que los adolescentes tienen de su comportamiento es muy similar a la expresada por sus compañeras, señalando que la conducta típica masculina en los contextos de ocio y consumo de alcohol es la violencia, motivada esta, tal y como relatan algunos de los participantes en el grupo focal, por una ingesta abusiva de esta sustancia y/o por peleas relacionadas con las relaciones de afecto:

Chica 6: Y se vuelven más agresivos de lo normal.

Chica 5: Sí, eso sí.

Chica 3: Celosos.

Chica 6: Sí, también. Y se meten con todo el mundo. Hay más broncas, que si empujones sin querer, no sé qué.

Chica 3: Bueno, más gallitos (GF03. Chicas 16-18 años).

Preceptora: ¿Y cuál creéis que puede ser el motivo de que haya bastantes peleas en las zonas de bares?

Chico 1: El alcohol.

Chico 2: Por el alcohol o por una chica.

Chico 1: El alcohol hace que te sulfures, cuando bebes no piensas y cuando te das cuenta ya te peleaste y todo. (GF04. Chicos 16-18 años)

En estos discursos se observa una relación entre agresividad y masculinidad que nuevamente remite a los tradicionales roles de género. Las mujeres perciben que los varones se pelean para demostrar su superioridad ante los demás, su masculinidad. Si bien esta agresividad masculina no es valorada de forma positiva por parte de las mujeres, de hecho, utilizan despectivamente el término "gallitos" para aludir a la acción masculina. Por su parte, los varones asumen la violencia como una conducta típica masculina, normalizada y aceptada. 
Sin embargo, hay dos cuestiones llamativas que conviene analizar, por un lado, los discursos de unas y otros se refieren a la violencia intrínseca de los varones, que se agudiza tras una ingesta excesiva de bebidas alcohólicas: "se vuelven más agresivos de lo normal", "el alcohol hace que te sulfures". Lo que indica que, a pesar de los avances sociales en contra de la violencia de género, la violencia masculina sigue constituyendo un tipo de conducta socialmente normalizada y percibida como tal por las y los adolescentes. Pero, además, cuando la citada violencia concurre con un consumo abusivo de alcohol parece que se diluye la intencionalidad y la responsabilidad masculina, lo hacen "sin querer", como plantean tanto el participante 1 como la participante 6 .

Por otro lado, en el discurso de los y las adolescentes hay referencias que o bien constituyen violencia de género o al menos violencia en las relaciones de afecto que, sin embargo, no es identificada como tal, sino únicamente como una cuestión de celos, como señala la adolescente 6 , o la mención "por una chica" del adolescente 2. Lo que de nuevo legitimaría el comportamiento violento masculino y remite a una interpretación de la violencia ligada a las relaciones afectivas propia de épocas pasadas en las que los celos y el carácter pasional justificaban la violencia masculina.

En contraposición a la expresión de emociones, que como se ha podido apreciar, es considerada una capacidad fundamentalmente femenina, las conductas agresivas ya no son privativas de los varones. En los discursos de los y las adolescentes aparecen ejemplos de agresividad femenina vinculada al consumo excesivo de alcohol. Pero nuevamente el significado que unas y otros otorgan a tales conductas difieren. En esta etapa del final de la adolescencia las mujeres identifican que la agresividad masculina tiene que ver con la construcción de la masculinidad tradicional, no con la identidad femenina, por eso cuando las mujeres ejercen violencia se las percibe como sujetos que van en contra de la norma, que rompen con los tradicionales roles de género:

Chica 7: Yo veo más hombres que mujeres, pero yo veo lo de los hombres porque quieren mostrar que eres más hombre, pero en cambio las mujeres a veces eso nos da mucho más igual, pero también las hay que tienen su carácter y su orgullo y también van diciendo: "Pues yo aquí, la que más".

Chica 8: Hay chorbas que son peores que los hombres. Chica 7: De hecho, una vez vi como una dejaba a un tío cao.

Chica 8: Ay ¡Qué fiera! Si estoy yo la animo.

Chica 7: No sé, eso también depende, pero normalmente suelen ser más los hombres, por eso de demostrar... (GF03. Chicas 16-18 años)
En estos discursos se puede observar cómo la agresividad femenina se percibe como un desafío a la feminidad tradicional. Las participantes en el grupo focal valoran de manera positiva el comportamiento de aquellas pocas que se atreven a utilizar la violencia, que interpretan como una especie de instrumento de autoafirmación personal, de superación de la tradicional pasividad y desvalorización impuesta a las mujeres: "las hay que tienen su carácter, su orgullo", incluso de empoderamiento: "Yo aquí, la que más". A pesar de ello, la expresión de sorpresa, e incluso de admiración, al relatar la pelea entre una mujer y un hombre observada en una ocasión, con resultado favorable a la primera, muestra que dicha conducta no está socialmente normalizada.

Este mismo relato aparece en los adolescentes, que también refieren un ejemplo de violencia femenina tras una ingesta excesiva de alcohol:

Chico 3: Puede parecer que puede ser más propio de chicos lo de pelearse, pero precisamente el año pasado, creo que tú estabas también.

Chico 4: Sí.

Chico 3: En la puerta del [mencionan un conocido bar nocturno], no sé si lo conocéis, hubo una chica que le lanzó un vaso a la cara a otra y la dejó sangrando y todo. (GF04. Chicos 16-18 años)

Paradójicamente los y las adolescentes apenas relatan las conductas agresivas habituales protagonizadas por varones, pero sí comentan cómo y cuándo vieron a una mujer golpear a un varón o lanzarle un vaso a la cara a otra. Hechos puntuales que, sin embargo, adquieren gran visibilidad precisamente porque se salen de las tradicionales normas de género, tal como algunas autoras han puesto de manifiesto al analizar procesos de transformación de los roles de género (Dema Moreno, 2008).

Por último, otra de las diferencias de género que aparece en los grupos focales tiene que ver con las formas de interacción física y virtual de los y las adolescentes. En los relatos de las chicas se pone de manifiesto su necesidad de interactuar y establecer relaciones sociales fuera del propio grupo de iguales, mientras que ellos prefieren la interacción dentro del mismo:

Chica 3: Por ejemplo, nosotras, yo muchas veces acabo sentada o mis amigas también, porque te duelen los pies. Los chicos no tienen ese problema, tienen la suerte, y bueno... Pero sí, siempre están como más, por lo menos en mi grupo, están, los chicos, ¿vale? están con nosotras, pero están como ahí a su rollo y nosotras ya somos más las que, pues bueno, vamos a saludar, vamos a no sé dónde, vamos a dar una vuelta. Ellos son más, eso, más estáticos, sí. Pero también be ben el triple. (GF03. Chicas 16-18 años) 
Los chicos coinciden en señalar que interactúan mayoritariamente con el grupo con el que salen, esgrimiendo en su discurso dos tipos de razones, por un lado y principalmente, destacan el valor del grupo que les acoge, que les ofrece un espacio de bienestar y de autonomía y, por otro, el consumo de alcohol, que les hace sentirse bien, por lo que no necesitan establecer relaciones sociales ni interactuar con otras personas ajenas al mismo:

Chico 1: Bueno, también bebes y estás con los amigos y porque te sientes en un ambiente cálido con tus amigos, te sientes cómodo y cuando ya estás un poco... que ya bebiste, te sientes bien, aunque hayas bebido... yo me entiendo es que no sé explicarlo. Me pasa con él y otro amigo que cuando estamos y ya si hemos bebido un poco...

Chico 3: Estamos en nuestra nube.

Chico 1: Se siente bien, que no hay nadie más alrededor nuestro.

Chico 3: Eso mismo.

Chico 1: Pero, y eso, ¿vale? Eso también nos gusta.

Chico 3: Eso, aún sin estar bebidos.

Chico 1: Sí, sin estar bebidos.

Chico 3: Estamos siempre a nuestra bola. (GF04. Chicos 16-18 años)

Es muy llamativo observar estos comportamientos diferenciados de varones y mujeres jóvenes, que nos remiten a una ruptura en los tradicionales roles de género. Frente a las formas de sociabilidad masculina tradicionales, en las que los varones son los que ocupan el espacio y se relacionan con otros grupos, en estos fragmentos se puede observar que son las adolescentes quienes establecen vínculos más allá del propio grupo de iguales, ocupando los diferentes espacios de los lugares de ocio. Por el contrario, los adolescentes relatan que permanecen en un mismo lugar, de forma estática, relacionándose únicamente con los integrantes de su grupo de iguales, una práctica que alude a los roles que tradicionalmente desempeñaban las mujeres en los espacios públicos.

En lo que se refiere al espacio virtual se encuentran pautas comparables. Ambos sexos coinciden en señalar diferencias respecto a la utilización de las redes sociales, y al papel que estas desempeñan en su ocio de fin de semana relacionado con el consumo de alcohol. Como se puede observar en los siguientes fragmentos, tanto unas como otros destacan que el uso de las tecnologías de la información y la comunicación por parte de las chicas está relacionado con la realización de fotos que posteriormente suben a alguna red social:

Chica 1: Yo creo que nunca he visto un chico con una cámara.

Chica 3: Ni yo. Las fotos subidas siempre son: las chicas un montón y los chicos cuatro.
Chica 1: Sí.

Chica 3: Y son de fútbol. (GF03. Chicas 16-18 años)

Chico 4: No salí yo nunca con una cámara de casa un sábado. Si hay fotos mías será porque me las sacan, pero no porque yo vaya sacando fotos a los demás.

Chico 1: Yo tampoco. Chico 1: Te encuentras una amiga y: "Ay, foto, foto, foto".

$(\ldots)$

Chico 4: En mi caso no, si las suben las suben otras personas... Además se puede ver: "varón 3 fotos, varón 3 fotos y mujer 35 fotos, varón 3 fotos”. (GF04. Chicos 16-18 años)

Los relatos de ambos grupos confluyen a este respecto, las adolescentes son las encargadas de tomar imágenes con las que narran sus salidas, mientras que los adolescentes no desarrollan esta práctica en la misma medida. En los discursos se observa que ellos identifican claramente esta práctica femenina como abusiva ("Te encuentras a una amiga y: Ay, foto, foto, foto”). Ellas, por su parte, hacen referencia a los tradicionales roles masculinos en el contenido de las fotos de los varones ("Son de fútbol").

Los varones, por el contrario, no utilizan las redes en la misma medida, por lo que realizan una crítica estereotipada desde el punto de vista del género, al uso que realizan las chicas de las TIC:

Chico 4: No sé, yo creo que, no sé si lo harán para ligar o no, pero a veces parece que hay grupos de amigas que más que salir a socializar, salen a sacarse fotos para Tuenti. A mí me da esa sensación.

Chico 1: Sí, sí.

Chico 4: Sí, no sé, cogen van y dicen: "Bueno, voy a exhibirme aquí un poco delante de la gente y luego voy a mi casa y subo las fotos que me hice esta tarde”. $\mathrm{Y}$ a veces es lo que hacen, eso, y nada más.

Chico 3: Foto, Tuenti. (GF04. Chicos 16-18 años)

En este discurso se observa cómo los adolescentes interpretan que el uso femenino de las TIC es abusivo y responde a una necesidad de recibir aprobación social. Consideran que las adolescentes se dedican a exhibir su imagen, e incluso entienden que dicha práctica constituye un fin en sí mismo (“A veces es lo que hacen, eso, y nada más”), como si no salieran a divertirse sino únicamente a sacarse las fotos, con el objetivo de ligar, que señala, aunque de forma dubitativa, el participante 4 .

\section{Etapa 3: Hacia un modo adulto de consumo de alcohol mediatizado por la identidad de género}

En esta última etapa, las mujeres de 19 a 24 años continúan asociando el ocio de fin de semana con salir de bares y con el consumo de bebidas alcohólicas, pero exponen 
que en esta edad ejercen un mayor control sobre su cuerpo y su conducta y, por tanto, realizan un consumo moderado, que no tiene como finalidad la búsqueda de la intoxicación etílica, como sucedía cuando eran más jóvenes:

Chica 1: Es lo que mis amigas y yo llamamos el "golpe de calor", que es que tú no vas a buscar... Yo sé que hay gente de mi edad, yo tengo 24, que sí, que sigue buscando cada fin de semana, sobre todo chicos, me tengo que emborrachar sí o sí. (GF05. Chicas 19-24 años)

Por el contrario, no perciben que los varones hayan experimentado dicho cambio con la edad, sino que siguen consumiendo en grandes cantidades, con el objetivo de emborracharse y perder el control:

Chica 1: Y cuando vas de Erasmus y tienes un grupo mixto sí que es verdad que los chicos tienen una actitud frente al alcohol que es totalmente distinta, es el hecho de: "Nosotros aguantamos más, luego bebemos más y es natural que acabemos como cubas rompiendo mobiliario urbano" y, bueno, pobres, tampoco todos, pero yo creo que en general es súper distinta la actitud frente al alcohol. (GF05. Chicas 19-24 años)

Como se puede observar, las jóvenes entienden que esta conducta está conectada con la necesidad que tienen los varones de reafirmar su masculinidad, lo que a su vez vinculan con una mayor tolerancia física al alcohol que, en cierto modo, como sucedía en el tramo de edad anterior, funciona como una forma de legitimar la mayor agresividad masculina. Las consecuencias de la violencia en este caso ya no se reducen a su implicación en peleas, sino que derivan en la comisión de actos vandálicos, como la destrucción de mobiliario urbano.

En los discursos masculinos también aparece la asociación entre ocio y consumo de alcohol, aunque ya no circunscriben esta actividad al fin de semana, aumentando, por tanto, el número de días a la semana que salen de bares y beben alcohol:

Chico 5: Según pinte... (risas todos) Es que para irte de fiesta no hace falta que sea fin de semana, o... pasarte un rato agradable por ahí, ¿no? Lo que es salir, yo qué sé... un día, un lunes, te puede apetecer, hacerte algo con unos amigos, vamos a tomar una copa y al final te has tomado cuatro. No sé, son espontáneos... momentos espontáneos... (GF06. Chicos 19-24 años)

En sus discursos, como en los de las mujeres jóvenes, también hacen referencia al consumo moderado de alcohol, pero en este caso asociado generalmente a los días de diario, mientras que los fines de semana es cuando suelen seguir realizando ingestas abusivas:
Chico 4: Fijos miércoles y viernes. Puede... la semana puede variar y puede ser además del miércoles y el viernes, jueves y sábados. Pero los jueves y los sábados pueden variar (risas todos). Pero... A ver... Tampoco es, salir hasta las tantas de la mañana, emborracharse todas esas veces. Los miércoles sales a tomar un par de cervezas a cierto bar que... pues bueno, tiene diferentes cervezas que no sean Mahou y Carlsberg... (GF06. Chicos 19-24 años)

En el consumo abusivo de alcohol la principal razón que esgrimen es la fuerte presión ejercida por el grupo de iguales, así como las dificultades que tienen para gestionar dicha presión:

Chico 3: Y también yo... sobre todo evitar... hacerte el valiente, en plan "¿A qué no tomamos dos Jäger seguidos o una de absenta? No sé qué...”

Chico 5: A que sí... (risas todos).

Chico 3: Bueno yo... Hay que tener la cabeza un poco fría para no caer en esas provocaciones que... jeje... Hay muchas a lo largo de la noche...

(risas todos)

Chico 4: Mentira. Mentira, caes..

Chico 2: Siempre caes... (risas todos). (GF06. Chicos 19-24 años)

En líneas generales, parece que comienza a fraguarse en esta franja de edad un cambio hacia un consumo de alcohol de menor riesgo en el caso de las mujeres jóvenes, pero no así en el de los varones. Desde estas nuevas pautas de consumo, las mujeres de 19 a 24 años realizan una crítica voraz y estereotipada desde el punto de vista del género al consumo de alcohol que realizan las personas de menor de edad, siendo especialmente incisivas en el caso de las adolescentes:

Chica 2: A ver, nosotras lo comentamos siempre, vivimos en el centro y cuando pasamos por ahí un sábado es que... es que es muy impactante ver a las niñas además con esos taconazos que no saben andar, que vale también lo hicimos nosotras, pero las ves y luego con unas borracheras y llorando. Que yo me acuerdo de un día que subí por [Menciona una conocida calle de bares] y es que realmente no vi a nadie pasándoselo bien, unos peleando, unas niñas llorando, yo que sé... Y yo decía, madre de Dios, es que esto...Y, sí, los ves muy pequeños, pero yo creo que es porque nosotros somos cada vez más mayores y entonces ves más la diferencia, pero igual me grabas a mí y estaba igual que ellos. (GF05. Chicas 19-24 años)

A través de estos relatos se observa claramente la existencia de una mayor sanción social para las chicas que 
realizan conductas alejadas del rol femenino, así como los estereotipos de género interiorizados que se utilizan para describir estas conductas. Parece incluso que es a través de los comportamientos de las chicas más jóvenes, que las mayores toman conciencia de la transgresión que, desde el punto de vista del género, realizaron en su adolescencia: "Vale, también lo hicimos nosotras, pero las ves y luego con unas borracheras y llorando", por lo que se produce una necesidad de establecer una diferencia clara entre sus acciones y las de las generaciones más jóvenes.

Los hombres jóvenes, por su parte, también critican el consumo de las adolescentes, que, al igual que sus compañeras, califican de abusivo, pero parece que atribuyen el fenómeno al cambio social. Desde su perspectiva, las adolescentes y jóvenes de hoy en día consumen en mayor proporción que las generaciones anteriores, superando la proporción de varones consumidores, lo que además consideran impropio desde el punto de vista de las relaciones de género:

Chico 6: Está cambiando mucho el chip, yo creo, en los últimos años, yo creo que de unos años para acá cambió todo mogollón. Las chicas incluso beben más que los chicos y luego actúan de manera muy distinta a como actuaba yo, por ejemplo, cuando tenía 16 años. Mi hermana mismamente, que es una cría, cambió totalmente a como era yo en su época. No sé, se emborrachan, llegan a casa que no se tienen de pie, cuando bueno, una vez, vale, pero cuando se hace varias veces... Y luego, no sé, vas por la calle y están tiradas por el suelo... y, no sé, no sé a qué se debe el cambio o que... (GF06. Chicos 19-24 años)

A través de estos discursos se observa nuevamente la extrema visibilización, ya explicada anteriormente, que se da a conductas que transgreden los roles de género. Al igual que ocurre en el caso de las mujeres que muestran conductas violentas, sigue sorprendiendo que las adolescentes consuman bebidas alcohólicas hasta llegar al extremo de una intoxicación etílica y que lo hagan en el espacio público. Según estos relatos parece que solo son las chicas las que se emborrachan y "andan tiradas por el suelo", cuando las diferencias en las prevalencias de borracheras entre unas y otros apenas alcanzan los tres puntos según la Encuesta ESTUDES realizada en 2014.

En definitiva, se aprecia en los discursos proporcionados por los y las jóvenes mayores de edad un acercamiento hacia el cumplimiento de los roles de género asignados, que desemboca en unas prevalencias y pautas de consumo de alcohol diferenciadas en función del sexo, así como en una necesidad de confirmar esos roles de género a través de la crítica y el distanciamiento con las conductas transgresoras que se producen en la adolescencia y la juventud temprana.

\section{Discusión}

Los resultados de este estudio revelan, en primer lugar, que existe una clara influencia de los roles de género en las pautas de consumo de alcohol durante la adolescencia y la primera etapa de la juventud. En segundo lugar, al haber tenido en cuenta las diferentes cohortes de edad establecidas para la configuración de los grupos focales, se ha podido comprobar cómo se manifiesta dicha influencia en tres etapas diferenciadas: el proceso de inicio y aprendizaje del consumo de alcohol, el desarrollo y la generalización de los consumos de riesgo en la etapa final de la adolescencia y, finalmente, la consolidación del consumo de alcohol en la primera etapa de la juventud, lo que previsiblemente marcará las pautas de consumo de estas personas en la edad adulta. Estos resultados reflejan también el precoz consumo de alcohol, las rutinas y relaciones sociales establecidas en torno a dicho consumo, el diferente valor de las mismas según el género, la evolución de las motivaciones a la hora de consumir alcohol y la reducida percepción de riesgo que se da en todo el proceso.

Los escasos estudios que incorporan una perspectiva de género en nuestro país señalan que los cambios en las pautas de consumo de alcohol de varones y mujeres revelan una serie de rupturas del sistema tradicional de género (Romo Avilés et al., 2015; Gómez Moya et al., 2010). Sin embargo, al no considerar la existencia de las tres etapas analizadas en este artículo, no pudieron identificar las diferencias de género que se producen en las diferentes fases de la adolescencia y de la juventud temprana, algo que solo aparece si se efectúa un análisis interseccional, como el llevado a cabo en este caso, y que puede tener especial relevancia a la hora de intervenir socialmente con este tipo de población, como se explicará más adelante.

Este estudio apunta a que durante la primera parte de la adolescencia los roles de género siguen estando claramente definidos y coincide con otras investigaciones al identificar que el consumo de alcohol y los contextos de ocio son prácticas y espacios masculinos, a los que tanto chicos como chicas acceden a través de figuras de mayor edad, generalmente varones con los que se tiene algún vínculo de amistad, familiar y/o afectivo (Romo Avilés et al., 2015; Gómez Moya et al., 2010; Lyons y Willott, 2008). En esta fase inicial, resulta llamativa la existencia de una práctica relacionada con el cuidado y la protección de los y las adolescentes en los espacios de ocio tras una ingesta abusiva de alcohol. Algunas autoras han identificado que el grupo de iguales funciona como una comunidad protectora que favorece la seguridad de sus integrantes cuando se llevan a cabo consumos de riesgo (Romo Avilés et al., 2015). Sin embargo, en esta investigación se ha podido observar que el rol de cuidado no corresponde al conjunto del grupo, sino fundamentalmente a aquellos individuos pertenecientes al mismo que practican un menor consumo. 
En el análisis realizado destaca, desde el punto de vista de género, el diferente valor social que se atribuye a dicha práctica cuando la realiza un varón o una mujer. En el caso de las mujeres se asocia a los tradicionales valores de cuidado, lo que produce una desvalorización de la conducta y de quien la realiza, mientras que, por el contrario, cuando son los varones quienes llevan a cabo las citadas labores de cuidado y control, su acción es reconocida y valorada por el grupo de iguales.

Los discursos analizados en este artículo concuerdan con los datos proporcionados por la Encuesta ESTUDES, que señala que al final de la adolescencia aparece una normalización y generalización del consumo abusivo de alcohol en ambos sexos, asociado al ocio de fin de semana, sobre todo en el caso de las chicas. Pero más importante aún que las prevalencias de consumo de dicha sustancia es el papel que el alcohol juega en la construcción de la identidad de chicos y chicas, puesto de manifiesto por la literatura especializada (Romo Avilés et al., 2015; Romo-Avilés et al., 2014; Lindsay, 2012; Rolfe et al., 2009; Peralta, 2007). Los resultados obtenidos en el análisis de los grupos focales muestran que los adolescentes construyen su masculinidad a partir de un consumo abusivo de esta sustancia y en dicho proceso la presión del grupo de iguales es central, particularmente en la incitación al consumo. Los discursos de ambos sexos vinculan asimismo la ingesta excesiva de bebidas alcohólicas y la violencia masculina, como expresión normalizada y aceptada de la masculinidad, en concordancia con el rol masculino tradicional.

En el caso de las adolescentes, sin embargo, se observa una subversión de los tradicionales roles de género femeninos. No sólo por la mayor prevalencia de consumo de alcohol que practican en este momento vital, tal como recoge la Encuesta ESTUDES, sino también por las formas de ocupación del espacio físico y virtual que relatan en los grupos focales. Frente a la tradicional división del espacio público-privado, asociado el primero a los varones y el segundo a las mujeres (Murillo, 1996), los discursos analizados refieren que las mujeres ocupan el espacio físico y virtual en mayor medida que los varones. Esta práctica se relaciona con cuestiones ligadas a la identidad de género, como la necesidad femenina de sentirse parte de una comunidad más amplia que el propio grupo, lo que proporciona a las jóvenes mayor seguridad en sus salidas nocturnas (Alcedo Rodríguez, Dema Moreno, Fontanil Gómez y Solís García, 2014). Asimismo, las adolescentes utilizan las citadas tecnologías para plasmar en imágenes sus salidas y divulgarlas en las redes sociales. Estas publicaciones les permiten mostrar públicamente su imagen, cuidada al detalle, y constituyen un elemento central en la construcción de la identidad femenina. De tal manera, que a través de las redes sociales muestran su participación en prácticas y espacios tradicionalmente masculinos, pero buscando en lo posible no comprometer su feminidad (Hutton, Griffin, Lyons, Niland y McCreanor, 2016).
Por último, si bien la violencia ejercida por los varones, tanto en el espacio público como privado, ha sido un tradicional objeto de estudio en las investigaciones con perspectiva de género, no ha sido hasta fechas recientes cuando la violencia femenina ha comenzado a ser analizada, destacando en el ámbito del ocio nocturno la investigación de Day, Gough y McFadden (2004). En los grupos focales, ambos sexos refieren algunos episodios violentos realizados por mujeres que son interpretados como una ruptura con los tradicionales roles de género, pero también sobre todo por parte de las mujeres como un modo de subversión e incluso como una forma de empoderamiento femenino. A pesar de que tales hechos son puntuales adquieren bastante visibilidad en los relatos, lo que podría ser interpretado como el comienzo de un proceso de cambio en los roles de género, aún no normalizado. Algunas autoras han puesto de manifiesto que precisamente cuando se dan procesos de cambio en los roles de género es frecuente observar una extrema visibilidad de aquellas conductas que se salen de la norma (Dema Moreno, 2008).

Finalmente, en la primera etapa de la juventud desaparece la transgresión característica de la etapa anterior y se observa un acercamiento por parte de las chicas hacia los roles de género asignados. En este momento vital, y de forma coincidente con la percepción de las personas participantes en los grupos focales, la Encuesta EDADES muestra que las mujeres comienzan a reducir las prácticas de riesgo relacionadas con el consumo de alcohol, mientras que los varones las mantienen y consolidan. Es también en este momento en el que aparecen con fuerza los discursos críticos, tanto de varones como de mujeres, con los comportamientos que se salen de las normas de género llevados a cabo por las adolescentes de la etapa anterior. A pesar de que las mujeres jóvenes en su adolescencia se hayan comportado de forma transgresora, los roles de género se encuentran tan sumamente arraigados que hay una necesidad manifiesta de romper con el comportamiento pasado y encauzarlo hacia el modelo socialmente aceptado. Y, por lo tanto, comienzan a perfilarse en esta etapa las pautas de consumo de alcohol que caracterizan a mujeres y varones adultos, con unas prevalencias mayores en los hombres en lo que se refiere a la ingesta de bebidas alcohólicas y a la diversa problemática asociada a un consumo nocivo (Observatorio Español de la Droga y la Toxicomanía, 2015).

Cabe señalar una vez más que la muestra utilizada para la realización de esta investigación no es estadísticamente representativa de la población adolescente y joven de España, ya que se ha llevado a cabo un muestro intencional, con el objetivo de conocer la influencia que ejercen los roles de género en el proceso de inicio y desarrollo del consumo de alcohol. Atendiendo a este objetivo, se han mencionado algunas cuestiones que aparecen en los discursos de adolescentes y jóvenes, sin profundizar en las mismas, como es el caso del significado de la violencia de género en contextos 
de ocio y, en particular, la vinculada al consumo de alcohol, que bien podrían perfilarse como futuras líneas de trabajo.

Como se ha tratado de mostrar a lo largo de este artículo, género y edad están interrelacionados, actuando de forma combinada. $\mathrm{Al}$ incorporar un enfoque interseccional al análisis se ha logrado comprender de forma muy exhaustiva los significados que las personas adolescentes y jóvenes atribuyen al consumo de alcohol, así como las conductas que desarrollan en relación con esta sustancia. Dicho enfoque no sólo es útil desde el punto de vista teórico, sino que podría ser considerado a la hora de intervenir socialmente en este ámbito, como también han puesto de manifiesto otras autoras (Jiménez Rodrigo y Guzmán Ordaz, 2011). En definitiva, para que las actuaciones, particularmente las preventivas, sean más eficaces, no solo han de tener en cuenta las relaciones de género, sino que es preciso que consideren además los diferentes momentos o etapas evolutivas por las que pasan las personas en su adolescencia y juventud.

\section{Reconocimientos}

Para la realización de este artículo se han utilizado los grupos focales realizados en el marco del "Estudio sobre el consumo de alcohol en adolescentes y jóvenes del Concejo de Oviedo", desarrollado a través de un convenio de colaboración entre el Ayuntamiento de Oviedo, la Fundación C.E.S.P.A.- Proyecto Hombre Asturias y la Universidad de Oviedo. Las autoras agradecen a las personas participantes en los grupos focales su ayuda, sin ellas no habría sido posible la realización de este artículo, así como la cesión de los datos al resto de colegas del equipo investigador y a las entidades promotoras del mismo.

\section{Conflicto de intereses}

Las autoras de este artículo declaran que no existe ningún potencial conflicto de intereses relacionado con la elaboración y publicación del mismo.

\section{Referencias bibliográficas}

Alcedo Rodríguez, M.A., Dema Moreno, S., Fontanil Gómez, Y. y Solís García, P. (2014). Estudio sobre el consumo de alcohol en adolescentes y jóvenes del Concejo de Oviedo. Oviedo: Ayuntamiento de Oviedo.

Ayuntamiento de Oviedo (2008). URBAN Iniciativa Urbana Oviedo 2007-2013. Recuperado de http:/ / www.dgfc.sepg. minhafp.gob.es/sitios/dgfc/es-ES/ipr/fcp0713/p/ iu0713pidu/Documents/ASTURIAS/Iniciativa_Urbana_Oviedo_2007-2013.pdf.

Bousoño Serrano, M., Al-Halabí, S., Burón, P., Garrido, M., Díaz-Mesa, E.M., Galván, G.,... Bobes, J. (2017). Substance use or abuse, internet use, psychopathology and suicidal ideation in adolescents. Adicciones, 29, 97-104. doi:10.20882/adicciones.811.

Colell, E., Sánchez-Niubò, A. y Domingo-Salvany, A. (2013). Sex differences in the cumulative incidence of substance use by birth cohort. International Journal of Drug Policy, 24, 319-325. doi:10.1016/j.drugpo.2012.09.006.

Davies, E. L., Martin, J. y Foxcroft, D. R. (2013). Young people talking about alcohol: Focus groups exploring constructs in the prototype willingness model. Drugs: Education, Prevention and Policy, 20, 269-277. doi:10.310 9/09687637.2012.726662.

Day, K., Gough, B. y McFadden, M. (2004). «Warning! Alcohol can seriously damage your feminine health»: A discourse analysis of recent British newspaper coverage of women and drinking. Feminist Media Studies, 4, 165183. doi:10.1080/1468077042000251238.

Day, K., Gough, B. y McFadden, M. (2003). Women Who Drink and Fight: A Discourse Analysis of Working-class Women's Talk. Feminism E Psychology, 13, 141-158. doi:1 $0.1177 / 0959353503013002878$.

De la Villa Moral Jiménez, M., Rodríguez Díaz, F. J. y Sirvent Ruiz, C. (2005). Motivadores de consumo de alcohol en adolescentes: análisis de diferencias inter-género y propuesta de un continuum etiológico. Adicciones, 17, 105-120.

Delgado, B., Bautista, R., Inglés, C. J., Espada, J. P., Torregrosa, M. S. y García-Fernández, J. M. (2005). Diferencias de género en el consumo de alcohol y tabaco de estudiantes de educación secundaria obligatoria. Salud y Drogas, 5, 55-65.

Dema Moreno, S. (2008). Las desigualdades de género en las ONG de desarrollo: discursos, prácticas y procesos de cambio. Revista Española de Investigaciones Sociológicas, 122, 119-144.

Díaz-Mesa, E. M., García-Portilla, P., Fernández-Artamendi, S., Sáiz, P. A., Bobes Bascarán, T., Casares, M. J. y Bobes, J. (2016). Diferencias de género en la gravedad de la adicción. Adicciones, 28, 221-230. doi:10.20882/adicciones.829.

Emslie, C., Lewars, H., Batty, G. D. y Hunt, K. (2009). Are there gender differences in levels of heavy, binge and problem drinking? Evidence from three generations in the west of Scotland. Public Health, 123, 12-14. doi:10.1016/j.puhe.2008.06.001.

Galán, I., González, M. y Valencia-Martín, J. L. (2014). Patrones de consumo de alcohol en España: un país en transición. Revista Española de Salud Pública, 88, 529-540. doi:10.4321/S1135-57272014000400007.

Gómez Moya, J., Arnal Gómez, A., Martínez Vilanova, A. M. y Muñoz Rodríguez, D. (2010). Mujeres y uso del alcohol en las sociedades contemporáneas. Revista Española de Drogodependencias, 3, 273-284.

Hutton, F., Griffin, C., Lyons, A., Niland, P. y McCreanor, T. (2016). «Tragic girls» and «crack whores»: Alcohol, 
femininity and Facebook. Feminism E Psychology, 26, 7393. doi:10.1177/0959353515618224.

Jiménez Rodrigo, M. L. y Guzmán Ordaz, R. (2011). Género y usos de drogas: dimensiones de análisis e intersección con otros ejes de desigualdad. Oñati Socio-Legal Series, 2, 77-96.

Lindsay, J. (2012). The gendered trouble with alcohol: Young people managing alcohol related violence. International Journal of Drug Policy, 23, 236-241. doi:10.1016/j. drugpo.2011.12.002.

Lyons, A. C. y Willott, S. A. (2008). Alcohol Consumption, Gender Identities and Women's Changing Social Positions. Sex Roles, 59, 694-712. doi:10.1007/s11199-0089475-6.

Measham, F. y Østergaard, J. (2009). The public face of binge drinking: British and Danish young women, recent trends in alcohol consumption and the European binge drinking debate. Probation Journal, 56, 415-434. doi:10.1177/0264550509346526.

Murillo, S. (1996). El mito de la vida privada: de la entrega al tiempo propio. Madrid: Siglo XXI, España Editores.

Observatorio Español de la Droga y la Toxicomanía (2017). Informe 2016. Alcohol, tabaco y drogas en España. Recuperado de http://www.pnsd.msssi.gob.es/profesionales/ sistemasInformacion/informesEstadisticas/pdf/2016_ INFORME_OEDT.pdf.

Observatorio Español de la Droga y la Toxicomanía (2017). EDADES 2015-2016. Encuesta domiciliaria sobre Alcohol y Drogas en España. Recuperado de http://www.pnsd. msssi.gob.es/profesionales/sistemasInformacion/sistemaInformacion/pdf/2015_EDADES_Informe_.pdf.

Organización Mundial de la Salud (2014). Global status report on alcohol and health, 2014. Ginebra: Organización Mundial de la Salud.

Organización Mundial de la Salud (2010). Estrategia mundial para reducir el uso nocivo del alcohol. Ginebra: Organización Mundial de la Salud.

Ortiz García, P. y Clavero Mira, E. (2014). Estilos de consumo de sustancias adictivas en función del género. Una aproximación desde el análisis de discurso. Acta Sociológica, 64, 121-144.

Peralta, R. L. (2007). College Alcohol Use and the Embodiment of Hegemonic Masculinity among European American Men. Sex Roles, 56, 741-756. doi:10.1007/s11199007-9233-1.

Roberts, S. C. M. (2012). Macro-level gender equality and alcohol consumption: A multi-level analysis across U.S. States. Social Science Ẽ Medicine, 75, 60-68. doi:10.1016/j. socscimed.2012.02.017.

Rolfe, A., Orford, J. y Dalton, S. (2009). Women, Alcohol and Femininity: A Discourse Analysis of Women Heavy Drinkers' Accounts. Journal of Health Psychology, 14, 326335. doi:10.1177/1359105308100217.
Romo Avilés, N., Marcos Marcos, J., Gil García, E., Marquina Márquez, A. y Tarragona Camacho, A. (2015). Bebiendo como chicos: consumo compartido de alcohol y rupturas de género en poblaciones adolescentes. Revista Española de Drogodependencias, 40,13-28.

Romo-Avilés, N., Meneses-Falcón, C. y Gil-García, E. (2014). Learning to be a Girl: Gender, Risks and Legal Drugs Amongst Spanish Teenagers. En T. Ortiz-Gómez y M. J. Santesmases (eds.) Gendered Drugs and Medicine. Historical and Socio-Cultural Perspectives (pp. 217-236). Londres y Nueva York: Routledge.

Sánchez-Gómez, M.C. (2004). Investigación cualitativa. En F. Salvador, J. L. Rodríguez y A. Bolívar (Dirs.), Dicciona rio enciclopédico de didáctica. Vol. II (pp.95-102). Málaga: Aljibe.

Secades Villa, R., López Núñez, C., Fernández Artamendi, S., Weidberg, S. y Fernández Hermida, J. R. (2013). Diferencias de género en la prevalencia de los trastornos por uso de alcohol del DSM-IV en adolescentes. Adicciones, 25, 260-268. 